\title{
Thermal patterning of superconducting films
}

\author{
M. Tortarolo ${ }^{\text {a }}$, H. Ferrari ${ }^{\text {a,* }}$, M. Marconi ${ }^{\mathrm{b}}$, V. Bekeris ${ }^{\text {a }}$ \\ a Laboratorio de Bajas Temperaturas, Facultad de Ciencias Exactas y Naturles, Universidad de Buenos Aires, \\ Buenos Aires 1428, Argentina \\ b Laboratorio de Electrónica Cuántica, Facultad de Ciencias Exactas y Naturles, Universidad de Buenos Aires, \\ Buenos Aires 1428, Argentina
}

\begin{abstract}
The transient shape control of a superconducting high $T_{\mathrm{c}}$ film by selective optical heating has been addressed in this work, using a non-conventional technique that combines the application of a pulsed magnetic field and the optical heating of the superconducting film by a synchronized pulsed laser. A zero field cooled (ZFC) film was partially protected by a mask before exposing it to the laser radiation. The temperature at the illuminated area rises causing very fast local lowering of the vortex pinning force or may even rise above $T_{\mathrm{c}}$. As a result, flux penetrates completely the heated area and an effective smaller sample remains in a superconducting critical state. Flux penetration is measured with a pick-up coil for different positions of the mask. Results are described within the critical state model for a strip, where the time integrated voltage signal is approximated to the flux difference between the initial ZFC sample and the final critical state of the "new" (cold) ZFC sample.
\end{abstract}

(C) 2004 Elsevier B.V. All rights reserved.

Keywords: Short time; Dc magnetization; Critical state

\section{Introduction}

In previous work, we reported on dc magnetization measurements $[1,2]$ on a HTS superconducting film in the $10^{-4} \mathrm{~s}$ time scale, well below the time resolution of conventional techniques $(\sim 1 \mathrm{~s})$. The zero field cooled (ZFC) superconducting film is held at a temperature below the irreversibility line, a pulsed transverse magnetic field, $H_{\mathrm{a}}$, is applied and after a controlled delay time, $t_{\mathrm{d}}$, a short laser pulse heats the whole sample above the irreversibility line, resulting in a sudden lowering of the pinning force on the vortex structure. Flux penetration is monitored via the transient voltage pulse induced in a pick up coil placed close to the sample. The time integrated photo-signal is the magnetic flux variation, which was found to be proportional to the effective

\footnotetext{
${ }^{*}$ Corresponding author. Address: Laboratorio de Bajas Temperaturas, Depto. de Física, Pabellón I Ciudad Universitaria, Buenos Aires 1428, Argentina. Fax: +54-11-4576-3357.

E-mail address: hferrari@df.uba.ar (H. Ferrari).
}

critical state film magnetization at $t_{\mathrm{d}}, M\left(t_{\mathrm{d}}\right)$ [2]. Sample shape control may be desirable for different applications. For this purpose, we selectively illuminate a ZFC film, positioning a mask to partially protect the film from radiation. As shown below, the monitored magnetic flux variation that occurs is a clear indication that the illuminated portion of the film is completely penetrated by the applied field in few nanoseconds, while the magnetic field redistributes in the protected part of the film. The final magnetic state can be well described by the critical state that corresponds to the ZFC "smaller" sample.

\section{Experimental}

The sample is a $\mathrm{GdBa}_{2} \mathrm{Cu}_{3} \mathrm{O}_{7-y}$ film $300 \mathrm{~nm}$ thick, dimensions $3.6 \times 7.0 \mathrm{~mm}^{2}$, zero field cooled to $75 \mathrm{~K} . T_{\text {irr }}$ at $H_{\mathrm{a}}=100$ Oe was $89 \mathrm{~K}$. A Nd(YAG) pulsed laser (FWHM: $10 \mathrm{~ns}, 10 \mathrm{~mJ} / \mathrm{cm}^{2}$ ) illuminated the sample which was partially protected with a rectangular mask, $5.2 \times 10.0 \mathrm{~mm}^{2}$, placed parallel to the film and close to the optical window of the cryostat. A micrometer screw 


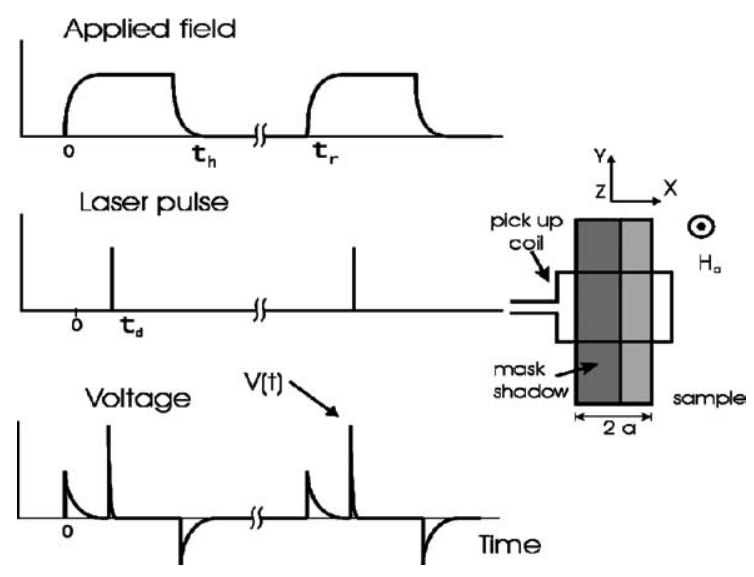

Fig. 1. Schematic time line of events. Inset: sample, mask and coil schematic.

controlled the position of the mask which was scanned across the film. The inset in Fig. 1 shows the mask shadow (in dark gray) on the sample (gray). The transverse pulsed magnetic field was provided by a primary coil inside the cryostat connected to a pulsed voltage source. The secondary coil consisted of one turn of copper wire (time constant below $5 \mathrm{~ns}$ ) wound closely on top of the film (see inset in Fig. 1). In Fig. 1, a schematic time line of events shows the repeated $\left(t_{\mathrm{r}} \sim 1 \mathrm{~s}\right)$ application of the pulsed field $\left(H_{\mathrm{a}} \sim 3000 \mathrm{~A} / \mathrm{m}\right)$ of duration $t_{\mathrm{h}} \sim 500 \mu \mathrm{s}$, whose rising edge $(\sim 3 \mu \mathrm{s})$ determines the time origin of the zero field cool (ZFC) state of the film. The laser pulse was triggered at $t_{\mathrm{d}}=60 \mu$ s selectively heating part of the film. The pick up coil voltage signal that followed, $V(t)$, was a pulse of several nanoseconds measured with a Tektronix TDS 3052, $500 \mathrm{MHz}$ and 5 GS/s oscilloscope.

\section{Results and discussion}

We describe our experiment as follows: the time scale of the field variation $(\sim 3 \mu \mathrm{s})$ is long compared to the vortex microscopic time scale, and a Bean profile for a ZFC strip of width $2 a$ in a transverse field [3] is already stationary at $t_{\mathrm{d}}=60 \mu \mathrm{s}$, when the laser pulse is triggered. In our simplified model, that neglects creep and heat diffusion effects, the illuminated area is heated above $T_{\text {irr }}$ and the rest of the sample remains at the initial low temperature. $H_{\mathrm{a}}$ fully penetrates the illuminated area of the film where pinning forces have suddenly dropped to zero, and flux redistributes in the cold (protected) portion of the sample according to the new boundary conditions. After thermal patterning, the final state should then correspond to a ZFC strip of width equal to

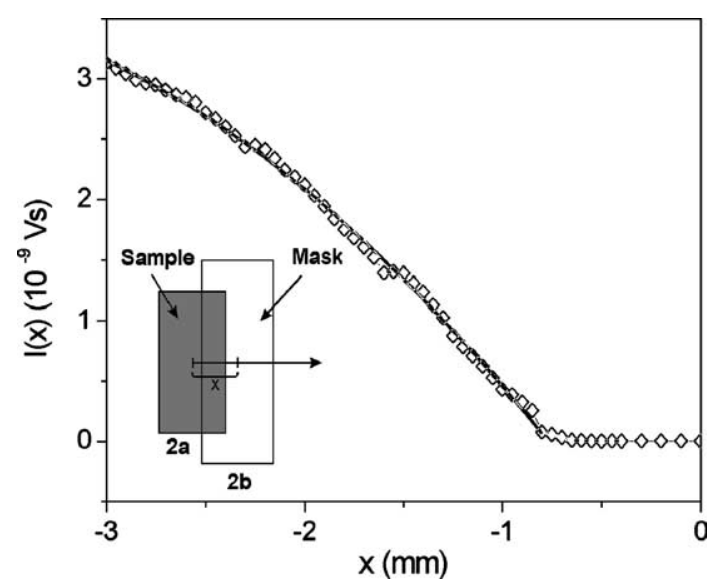

Fig. 2. Measured (open symbols) and calculated (heavy full line) time integrated voltage signals as a function of the position of the mask as indicated in the inset.

$(a+b)-|x|$ (the size of the mask shadow), where $2 b$ is the mask width and $x$ is the mask midpoint position relative to the sample midpoint (see lower inset in Fig. 2 where for clarity the coil is not shown). Fig. 2 shows in full line our calculations of flux variation between the described initial and final states. Symbols are the measured $I=\int V(t) \mathrm{d} t$. Our fitting parameters are the critical current density at $75 \mathrm{~K}$, which resulted $J_{\mathrm{c}}=5 \times 10^{9}$ $\mathrm{A} / \mathrm{m}^{2}$ in accordance with reported values [2], and the sample width $2 a=(3.6 \pm 0.2) \mathrm{mm}$ in good agreement with our sample dimension. In future work issues as the effect of creep [4] and thermal diffusion are to be addressed to attain a better understanding of "thermal patterning".

\section{Acknowledgements}

This work was supported by: UBACYT X181, X71 and CONICET PID 4634.

\section{References}

[1] S.O. Valenzuela, H. Ferrari, V. Bekeris, M.C. Marconi, J. Guimpel, F. de la Cruz, Rev. Sci. Instrum. 69 (1998) 251.

[2] S.O. Valenzuela, H. Ferrari, V. Bekeris, J. Guimpel, F. de la Cruz, Rev. Mex. Fís. 44 (1998) 193.

[3] E.H. Brandt, M. Indenbom, Phys. Rev. B 48 (1993) 12893.

[4] Calculations including creep see for e.g. E.H. Brandt, Phys. Rev. B 49 (1994) 9024;

H. Ferrari, D. Ibaceta, V. Bekeris, E. Calzetta, L. Correra, Cond-Mat 0011347, (2000). 